\title{
Advocating lifestyle modification to reduce risk of stone recurrence
}

\author{
Sero Andonian, MD, MSc, FRCSC, FACS
}

McGill University, Montreal, QC, Canada

Cite as: Can Urol Assoc J 2016;10(7-8):239-40. htrp://dx.doi.org/10.5489/cuaj.4001

See related article on page 234 .

$\mathrm{T}$ he authors have prospectively assessed stone recurrence in 110 participants using body mass index (BMI), fasting serum leptin and adiponectin, and proportion of visceral adipose tissue (\%VAT) obtained on axial computed tomography (CT) scans. ${ }^{1}$ Since BMI does not differentiate between peripheral and central obesity, the authors used elevated \%VAT as an indicator for central obesity. While $\mathrm{BMI}$ and obesometric serum hormones did not correlate with recurrence, elevated \%VAT was independently predictive of urolithiasis recurrence among initial stone formers (hazard ratio $[H R] 4.53$ ), but not among recurrent stone formers. ${ }^{1}$

This is a very nice prospective study providing evidence for central obesity (elevated \%VAT) correlating with stone recurrence. In addition to central obesity, metabolic syndrome traits also include raised triglycerides, reduced highdensity lipoprotein (HDL) cholesterol, raised blood pressure, and raised fasting plasma glucose. ${ }^{2}$ Previous studies have shown that patients with three or more metabolic syndrome traits were associated with higher prevalence of urolithiasis. ${ }^{2}$ Furthermore, patients with metabolic syndrome have been shown to have significantly higher stone recurrence post-percutaneous nephrolithotomy when compared with controls $(41.9 \%$ vs. $18.9 \%$; $p=0.003){ }^{3}$

In addition to the explanations provided by the authors for the lack of correlation of elevated \%VAT with stone recurrence in recurrent stone formers, I would like to propose these two hypotheses. Although the authors excluded patients with residual stone burden, it is possible that recurrent stone formers may have had clinically insignificant residual fragments that contributed to stone recurrence rather than stone recurrence being attributed solely to the elevated \%VAT and metabolic syndrome. In addition, as the authors mention, stone analyses and metabolic stone workup were not available for the study population. It is possible that recurrent stone formers may have had other metabolic abnormalities, such as primary hyperparathyroidism and distal renal tubular acidosis, unrelated to the elevated \%VAT and metabolic syndrome. These may have contributed to the recurrence of stones rather than obesometric parameters, such as elevated \% VAT.

What does this all mean? As the body of knowledge regarding metabolic syndrome correlating with urolithiasis grows, are we going to screen patients presenting with urolithiasis for metabolic syndrome traits by measuring waist circumference, waist-to-hip ratio, and \%VAT? I don't think it is feasible for the practicing urologist to invest in such meticulous measurements of central obesity to determine how many metabolic syndrome traits each patient has. However, the lifestyle modification interventions that we advise our patients based on the latest Canadian Urological Association guideline (smoking cessation, increasing exercise, increasing hydration, and reducing intake of salt and animal protein) will not only reduce risk of stone recurrence, but have also been shown to be effective in resolving metabolic syndrome and reducing severity of its related abnormalities. ${ }^{4-7}$ Therefore, we can exercise our CanMEDS health advocate roles and advise urolithiasis patients with central obesity to follow these lifestyle modifications.

Competing interests: The author reports no competing personal or financial interests.

\section{References}

1. Bos D, Dason S, Matsumoto ED, et al. A prospective evaluation of obesometric parameters associated with renal stone recurrence. Can Urol Assoc J 2016;10:234-8. http://dx.doi.org/10.5489/cuai.3876

2. Wong Y, Cook P, Roderick P, et al. Metabolic syndrome and kidney stone disease: A systematic review of literature. J Endourol 2016;30:246-53. http://dx.doi.org/10.1089/end.2015.0567

3. Akman T, Binbay M, Erbin A, et al. The impact of metabolic syndrome on long-term outcomes of percutaneous nephrolithotomy (PCNL). BJU Int 2012;110:E1079-83. http://dx.doi.org/10.1111/i.1464410X.2012.11548.x 
Andonian

4. Soueidan $M$, Bartlett SJ, Noureldin YA, et al. Leisure time physical activity, smoking and risk of recent symptomatic urolithiasis: Survey of stone clinic patients. Can Urol Assoc J 2015;9:257-62. http://dx.doi.org/10.5489/cuaj.2879

5. Paterson R, Fernandez A, Razvi H, et al. Evaluation and medical management of the kidney stone patient. Can Urol Assoc J 2010;4:375-9. http://dx.doi.org/10.5489/cuaj.10166

6. Yamaoka K, Tango T. Effects of lifestyle modification on metabolic syndrome: A systematic review and meta-analysis. BMC Med 2012;10:138. http://dx.doi.org/10.1186/1741-7015-10-138
7. Andonian S, Elkoushy MA. Preventing stones and managing them once you get them. In. The Consumer's Handbook of Urological Health. Ed. Brock G. Canadian Urological Association. Dorval, QC 2013; 250-267.

Correspondence: Dr. Sero Andonian, Associate Professor of Urology, McGill University Health Centre, Montreal, QC, Canada; sero.andonian@muhc.mcgill.ca
McMaster

University

HEALTH SCIENCES

\section{Academic Urologist}

McMaster University and Hamilton Health Sciences are seeking an academic urologist. Hamilton Health Sciences serves a catchment area of more than 1.4

million residents providing comprehensive care across the sites of Hamilton Health Sciences. The Urology Division provides tertiary and quaternary level care to patients from other regions of the province and plays an essential role in the support of regional programs including gynecological oncology, colorectal surgery and surgical oncology, renal disease and transplantation. The Urology Division together with the Department of Surgery, McMaster University is a fully accredited Royal College of Physicians and Surgeons of Canada training program in Urology with full involvement in undergraduate, post-graduate and continuing medical education programs.

The successful candidate will be based at the Hamilton General Hospital and will be expected to demonstrate experience and training in neuro-urology, urodynamics and female pelvic floor reconstruction. In addition, it is expected that that the candidate will be able to participate and lead transitional care of adolescents with ongoing complex urologic care needs.

The successful applicant's clinical skill set must include facility with general urology for the purposes of regional call duties. He/she will be expected to show evidence of interpersonal and collaborative skills and initiative to further advance the clinical and academic productivity of the regional urology service. A full time appointment at the appropriate level in the Department of Surgery, Faculty of Health Sciences, McMaster University is required and will be available to the successful applicant. A commitment and demonstrated interest in surgical education at all levels is essential, as well as active participation in research programs and academic initiatives of the Division of Urology.

Applicants must have fellowship training in neuro-urology, urodynamics and pelvic floor reconstruction. Candidates must have certification by the Royal College of Physicians and Surgeons of Canada, or equivalent, and be eligible for licensure in the Province of Ontario. Preference will be given to applicants with interest in clinical trials in urology and evidence based surgery.

All qualified candidates are encouraged to apply. However, Canadian citizens and permanent residents will be considered first for this position. To comply with the Government of Canada's reporting requirements, the University is obliged to gather information about applicants' status as either Permanent Residents of Canada or Canadian citizens. Applicants need not identify their country of origin or current citizenship; however, all applications must include one of the following statements:

"Yes, I am a citizen or permanent resident of Canada" or "No, I am not a citizen or permanent resident of Canada"

Applications must include an up-to-date curriculum vitae, and a description of previous academic experience, as well as of specialty training. This application will remain open until the position is filled. Applications are to be submitted to

Dr. Susan Reid, Professor and Chair, Faculty of Health Sciences, McMaster University

B3-155 Juravinski Hospital \& Cancer Centre

711 Concession Street

Hamilton, ON L8V 1 C3

Email: painebr@mcmaster.ca 